\title{
Marked Elevation of Serum Hyaluronan Levels in Collagenofibrotic Glomerulopathy
}

\author{
Shunsuke Goto ${ }^{1}$, Kentaro Nakai ${ }^{1}$, Jun Ito ${ }^{1}$, Hideki Fujii ${ }^{1}$, Kazuyuki Tasaki ${ }^{2}$, \\ Toshiaki Suzuki ${ }^{3}$, Kei Fukami ${ }^{4}$, Shigeo Hara ${ }^{5}$ and Shinichi Nishi ${ }^{1}$
}

\begin{abstract}
Collagenofibrotic glomerulopathy is a rare glomerular disease characterized by the massive deposition of type III collagen in mesangial and subendothelial spaces. We observed markedly increased serum hyaluronan levels in patients with collagenofibrotic glomerulopathy; levels in three patients were more than 1,000-times greater than the normal upper limit. However, one kidney transplant patient had normal serum hyaluronan levels. We found that serum levels and activities of the enzyme hyaluronidase were normal, and hyaluronan was not markedly deposited in the mesangial or subendothelial spaces. Our findings suggest that serum hyaluronan levels may be a specific diagnostic marker of collagenofibrotic glomerulopathy, and kidney transplantation may alleviate marked increases in serum hyaluronan.
\end{abstract}

Key words: collagenofibrotic glomerulopathy, hyaluronan, hyaluronidase

(Intern Med 53: 1801-1804, 2014)

(DOI: 10.2169/internalmedicine.53.2412)

\section{Introduction}

Collagenofibrotic glomerulopathy is a rare glomerular disease characterized by the massive deposition of type III collagen fibers in mesangial and subendothelial spaces and increased serum procollagen type III peptide levels; it is suspected to be a hereditary disease (1). Although about 40 cases have been reported in English articles, the pathogenesis of this glomerulopathy has not been elucidated.

Hyaluronan is a glycosaminoglycan found in the extracellular spaces of most tissues. Serum levels in middle-aged individuals range from $10-100 \mathrm{ng} / \mathrm{mL}$ (2). Although serum hyaluronan levels are known to be increased in several conditions, such as liver disease or inflammatory disease, and they can be as high as $2,000 \mathrm{ng} / \mathrm{mL}$ (2). We measured the serum hyaluronan levels in one patient with collagenofibrotic glomerulopathy and found that they were $>100,000$ $\mathrm{ng} / \mathrm{mL}$, although other symptoms of liver disease and inflammation were absent. We report serum hyaluronan levels in the patient, her son, and three other collagenofibrotic glomerulopathy patients. In addition, we investigated serum hyaluronidase enzyme levels and activities, as well as the deposition of hyaluronan in glomerular lesions.

\section{Case Report}

The brief medical histories of four patients are described below. Descriptions of patients three and four have been published previously $(3,4)$.

Patient 1 was a 74-year-old Japanese female who was referred to our hospital after suffering from protracted leg edema for five years. Her serum creatinine levels were 1.57 $\mathrm{mg} / \mathrm{dL}$, urinalysis revealed no hematuria, and urinary protein excretion was $4.3 \mathrm{~g} / \mathrm{day}$. Following renal biopsy, she was diagnosed with collagenofibrotic glomerulopathy, and her serum procollagen type III peptide level was $7.0 \mathrm{U} / \mathrm{mL}$. She was subsequently treated with antihypertensive medications, and after 2 months, her serum creatinine level was $1.45 \mathrm{mg} /$ $\mathrm{dL}$ and her urinary protein-to-creatinine ratio was 9.7.

\footnotetext{
${ }^{1}$ Division of Nephrology and Kidney Center, Kobe University Graduate School of Medicine, Japan, ${ }^{2}$ Department of Nephrology, Saiseikai Niigata Daini Hospital, Japan, ${ }^{3}$ Pediatrics, Niigata University Graduate School of Medicine and Dental Sciences, Japan, ${ }^{4}$ Division of Nephrology, Department of Medicine, Kurume University School of Medicine, Japan and ${ }^{5}$ Department of Diagnostic Pathology, Kobe University Graduate School of Medicine, Japan

Received for publication December 29, 2013; Accepted for publication January 30, 2014

Correspondence to Dr. Shinichi Nishi, snishi@med.kobe-u.ac.jp
} 
Table. Demographic and Laboratory Data on Measurement of Serum Hyaluronan Levels

\begin{tabular}{|c|c|c|c|c|c|c|}
\hline & Age & Sex & $\begin{array}{l}\text { Hyaluronan } \\
(\mathrm{ng} / \mathrm{mL})\end{array}$ & $\begin{array}{l}\text { Hyaluronidase-1 } \\
(\mathrm{ng} / \mathrm{mL})\end{array}$ & $\begin{array}{c}\text { Hyaluronidase-2 } \\
\text { (ng/mL) }\end{array}$ & $\begin{array}{l}\text { Creatinine } \\
(\mathrm{mg} / \mathrm{dL})\end{array}$ \\
\hline Normal range & & & $<50$ & & & \\
\hline Control $(\mathrm{N}=12)$ & & & $24 \pm 19$ & $3.05 \pm 2.41$ & $0.98 \pm 0.68$ & \\
\hline [range] & & & {$[<10-69.1]$} & {$[0.56-7.12]$} & {$[0-2.46]$} & \\
\hline Patient 1 & 74 & $\mathrm{~F}$ & $112,000.0$ & 5.16 & 1.83 & 1.34 \\
\hline Patient 2 & 72 & M & $53,798.0$ & 1.18 & 0.89 & Hemodialysis \\
\hline Patient 3 & 79 & $\mathrm{~F}$ & $71,800.0$ & 2.30 & 6.36 & 1.30 \\
\hline Patient 4 & 28 & $\mathrm{~F}$ & 21.0 & 2.51 & 0.62 & 0.91 \\
\hline Son of Patient 1 & 53 & M & 80.7 & 4.82 & 0.53 & 0.81 \\
\hline
\end{tabular}

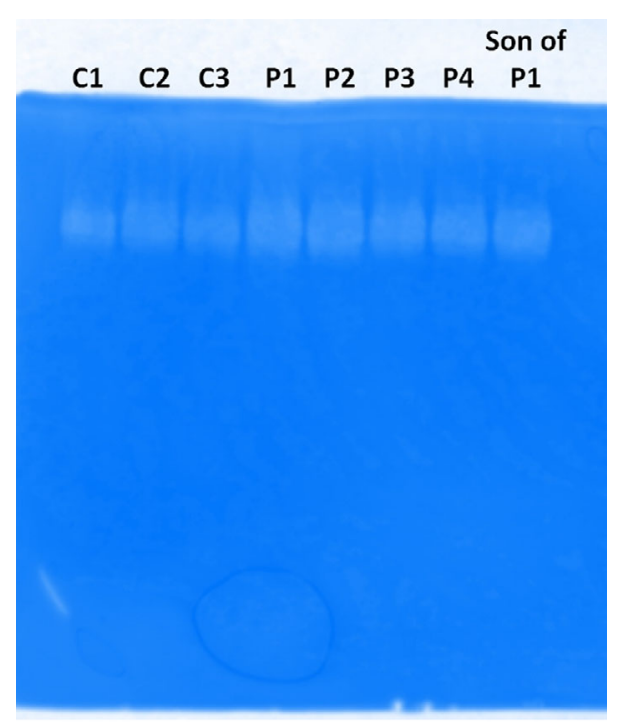

Figure 1. Zymography measurements of serum hyaluronidase activity. Hyaluronan was stained using Alcian blue. Hyaluronidase activities are observed as white bands, which indicate comparable activity in patients and controls. $C$ : control, $P$ : patient

Patient two was a 54-year-old Japanese male who was referred to our hospital with persistent proteinuria for four years. His serum creatinine level was $0.8 \mathrm{mg} / \mathrm{dL}$. Urinalysis revealed no hematuria, and his urine protein excretion was $0.6 \mathrm{~g} /$ day. Following renal biopsy, he was diagnosed with collagenofibrotic glomerulopathy, and his serum procollagen type III peptide level was $2.9 \mathrm{U} / \mathrm{mL}$. Following conservative therapy, his proteinuria persisted, and his renal function deteriorated. He required hemodialysis at the age of 65 years. The patient's brother also had proteinuria, was diagnosed with collagenofibrotic glomerulopathy following a renal biopsy at the age of 53 years, and subsequently died of esophageal cancer six years later.

Patient three was a 79-year-old Japanese female who was admitted to our hospital for leg edema. Her serum creatinine level was $0.93 \mathrm{mg} / \mathrm{dL}$, and her urinary protein-to-creatinine ratio was 10.2. The patient's urinalysis revealed microscopic hematuria, and she was diagnosed with collagenofibrotic glomerulopathy following renal biopsy; her serum procollagen type III peptide level was $12.0 \mathrm{U} / \mathrm{mL}$. After admission, she received conservative therapy. Subsequently, her serum creatinine levels stabilized, and her urinary protein excretion decreased.

Patient four was a 6-year-old Japanese female who was referred to our hospital for proteinuria and hematuria. Her urinary protein excretion was 3-4 g/day, and she was diagnosed with collagenofibrotic glomerulopathy following a renal biopsy. Her serum procollagen type III peptide level was 6.1 U/mL. Her renal function subsequently deteriorated despite steroid, cyclosporine, cyclophosphamide, low-density lipoprotein (LDL)-apheresis, anticoagulant, and antiplatelet drug therapies. At the age of 13 years, she underwent peritoneal dialysis, and she received an ABO-compatible kidney transplantation from her father at age 18. She was subsequently treated with tacrolimus, methylprednisolone, and basiliximab, and her renal function was stable with no proteinuria detected in the urinalysis. Her serum procollagen type III peptide levels had decreased to $1.1 \mathrm{U} / \mathrm{mL} 10$ years after transplantation.

Table presents the serum hyaluronan and hyaluronidase levels from all four patients and the son of patient one who did not have any apparent disease and had a urinary proteinto-creatinine ratio of 0.1 . Patients one and three were examined one month and two years after renal biopsy, respectively. Patient two was examined seven years after the initiation of hemodialysis, and patient four was examined 10 years after transplantation. The serum hyaluronan levels of patients one, two, and three were markedly increased, but that of patient four, who had received a kidney transplant was within the normal range. The serum hyaluronan levels in the son of patient one were observed to have slightly increased.

Among several types of mammalian hyaluronidases, hyaluronidase- 1 and -2 predominate in human serum. Because the normal ranges of serum hyaluronidase- 1 and -2 levels are unclear, we compared the levels of these enzymes in the four patients with those in 12 healthy controls. The serum hyaluronidase-1 and -2 levels of all patients were comparable to those of controls. The zymograpy measurements of serum hyaluronidase activities were similar between patients and controls and are presented in Fig. 1.

To further investigate hyaluronan deposition, renal biopsy tissue from patient one was processed with a colloidal iron stain before and after hyaluronidase digestion. Light microscopy revealed poorly stained global mesangial expansions 


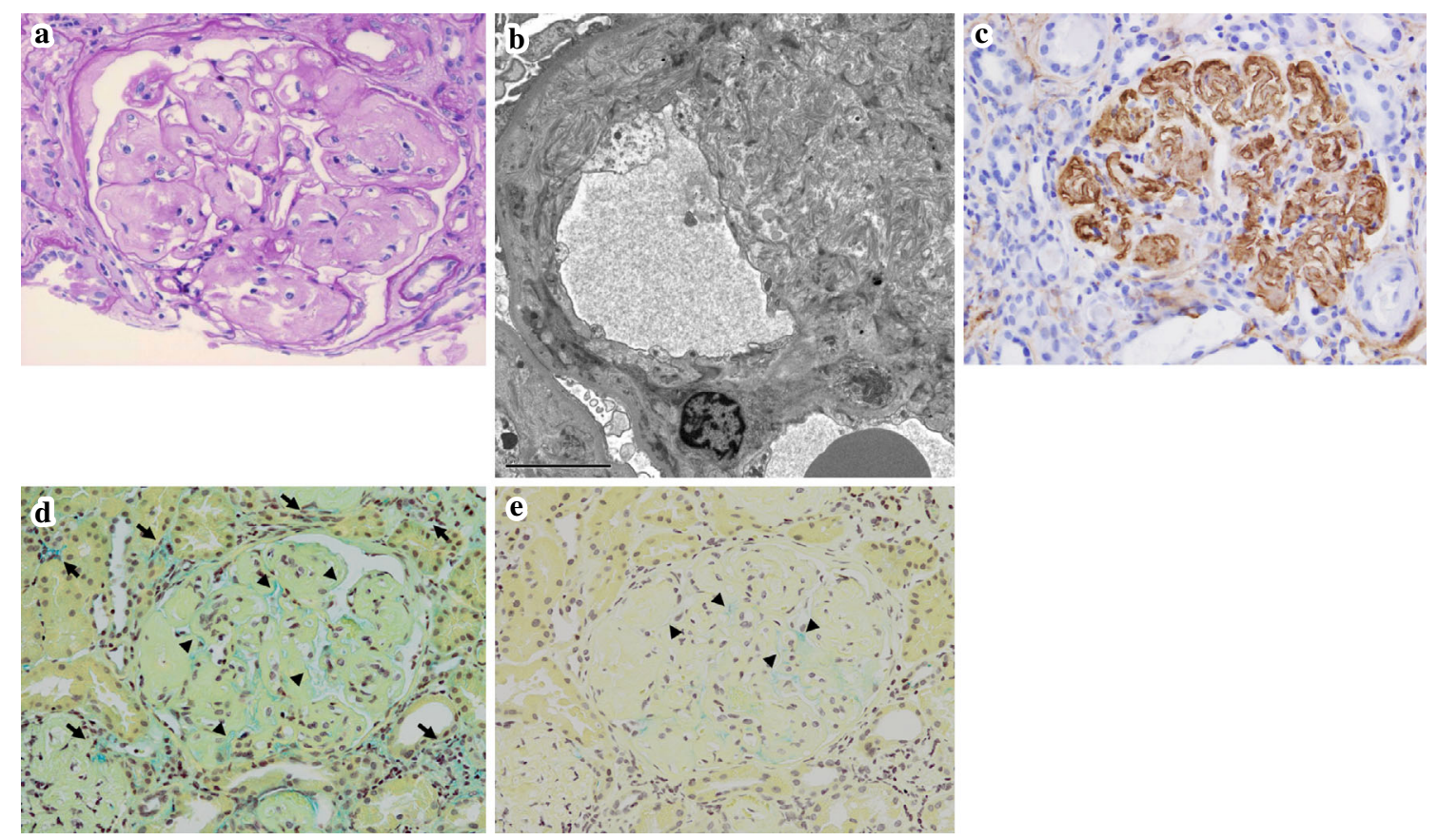

Figure 2. Histology findings in patients with collagenofibrotic glomerulopathy. a: Periodic acidSchiff-stained renal tissue from patient one. Lobular patterns with markedly increased mesangial matrix were observed in all glomeruli. b: Electron microscope images of glomeruli stained with tannic acid. Massive deposition of collagen fibers was observed in mesangial matrixes and subendothelial spaces. c: Immunohistological labeling of type III collagen showed diffusely positive areas in expanded mesangial matrices and capillary loops. d, e: Colloidal iron-stained renal tissues before and after hyaluronidase digestion, respectively. Mesangial and subendothelial spaces were not significantly stained with colloidal iron compared with the interstitia (arrows) and glomerular capillary surfaces (arrow heads). Hyaluronidase digestion abolished interstitium staining and partially attenuated capillary loop staining.

with lobulation (Fig. 2a). The expanded mesangial and subendothelial spaces exhibited massive deposition of type III collagen (Fig. 2b, c), but the glomeruli showed no evidence of marked hyaluronan deposition (Fig. 2d, e).

\section{Discussion}

The pathogenesis of collagenofibrotic glomerulopathy remains poorly characterized. The present cases exhibited markedly increased serum hyaluronan levels. Various diseases, such as liver disease, rheumatoid arthritis, sepsis, renal failure, and cancer (2), are associated with mean serum hyaluronan levels of up to $2,000 \mathrm{ng} / \mathrm{mL}$. With the exception of the renal transplant case (patient 4 ), serum hyaluronan levels were between 53,000 and $112,000 \mathrm{ng} / \mathrm{mL}$ in all of the patients described here, thus suggesting that serum hyaluronan levels are significantly involved in the pathogenesis of collagenofibrotic glomerulopathy.

In this study, the renal transplant patient with collagenofibrotic glomerulopathy had serum hyaluronan levels within normal limits. Although we did not measure serum hyaluronan levels prior to renal transplantation, intensive immunosuppressive therapy and the donated kidney may have strongly suppressed hyaluronan production or increased its degradation. Previous reports described that serum hyaluro- nan levels in patients receiving hemodialysis were higher compared with those in healthy controls and were up to 20 times the upper limit of the normal range $(5,6)$. Therefore, improvements of kidney function may not be sufficient to normalize serum hyaluronan levels in renal transplant patients with collagenofibrotic glomerulopathy.

Hyaluronidase is responsible for the degradation of hyaluronan (7), and six hyaluronidase genes have been identified in humans. Among these enzymes, hyaluronidase-1 and -2 degrade the majority of hyaluronan (7). In the present patients, serum hyaluronidase activity and hyaluronidase- 1 and -2 levels were not lower than those in healthy controls. In addition, mutation of the gene encoding HYAL1 in humans reportedly results in short stature, generalized cutaneous swelling, transiently painful soft tissue masses over articular surfaces, and bilateral joint effusion $(8,9)$. However, we did not observe these symptoms in patients with collagenofibrotic glomerulopathy. Moreover, renal dysfunction was not reported in patients with HYAL1 gene mutations. Taken together, these observations indicate that hyaluronidase is probably not involved in the marked increase in serum hyaluronan in patients with collagenofibrotic glomerulopathy.

Hyaluronan is synthesized by plasma membrane hyaluronan synthases (HAS) (10), and increased HAS activity may 
markedly increase serum hyaluronan in patients with collagenofibrotic glomerulopathy. However, we did not measure HAS activity in the present study, and this remains to be examined in patients with collagenofibrotic glomerulopathy.

We hypothesized that a marked increase in serum hyaluronan could lead to its deposition in the glomerulus. Therefore, we evaluated hyaluronan deposition with colloidal iron staining before and after hyaluronidase digestion. Colloidal iron staining is widely used to detect negatively charged surface matrices, and the interstitium generally contains large quantities of negatively charged glycosaminoglycans, such as hyaluronan (11). Moreover, glomerular capillary surfaces are covered by podocytes, which have anionic glycocalyx layers (12). Therefore, both the interstitium and glomerular capillary walls are normally stained by colloidal iron, and hyaluronidase digestion abolishes this staining in the interstitium but not in the capillary loops. We did not observe significant colloidal iron staining within glomeruli compared with that in the interstitium and capillary surfaces of patient one. Furthermore, the interstitial colloidal iron staining of kidney tissue did not differ between patient one and normal controls (data not shown), indicating that hyaluronan deposition is not markedly increased in kidney tissue from patients with collagenofibrotic glomerulopathy. It is unclear why hyaluronan was not significantly deposited within glomeruli despite markedly elevated serum hyaluronan levels. Nikolic-Paterson et al. demonstrated hyaluronan deposition in rat anti-Thy-1 nephritis and showed that type 1 transmembrane glycoprotein CD44 colocalized with hyaluronan deposition (13). Therefore, CD44 may be necessary for hyaluronan deposition. Further studies are needed to elucidate why significant hyaluronan deposition was not detected in the capillary loops in the patients described here.

The existence of sibling cases suggests that collagenofibrotic glomerulopathy may be an autosomal recessive genetic disease (1). Patient two's brother also had the disease, and the serum hyaluronan levels in the son of patient one were slightly above the normal range. Although we only performed appropriate measurements in one case, changes in the expression or function of hyaluronan-related genes may underlie the pathogenesis of collagenofibrotic glomerulopathy.

Although not all subjects with elevated serum hyaluronan have kidney disease, marked increases in hyaluronan may damage kidney function because hyaluronan can increase proteoglycan synthesis in mesangial cells (14). Further studies are necessary to investigate whether massive amounts of hyaluronan induce kidney disease.

In conclusion, we identified markedly increased serum hyaluronan levels in patients with collagenofibrotic glomerulopathy. Although the underlying mechanisms remain unclear, increased serum hyaluronan levels maybe a useful diagnostic marker of collagenofibrotic glomerulopathy.
The authors state that they have no Conflict of Interest (COI).

\section{Acknowledgement}

We thank Kayo Tubota for providing valuable technical assistance. This study was supported in part by a Grant-in-Aid for Progressive Renal Disease Research, Research on Rare and Intractable Disease, and Research on Amyloidosis from the Ministry of Health, Labour and Welfare of Japan and Grant-in-Aid issue number 24591199 from the Ministry of Education, Culture, Sports, Science and Technology of Japan.

\section{References}

1. Alchi B, Nishi S, Narita I, Gejyo F. Collagenofibrotic glomerulopathy: clinicopathologic overview of a rare glomerular disease. Am J Kidney Dis 49: 499-506, 2007.

2. Laurent TC, Laurent UB, Fraser JR. Serum hyaluronan as a disease marker. Ann Med 28: 241-253, 1996.

3. Fukami K, Yamagishi S, Minezaki T, Nishi S, Hisano S, Okuda S. First reported case of collagenofibrotic glomerulopathy with a fullhouse pattern of immune deposits. Clin Nephrol 81: 290-295, 2014.

4. Suzuki T, Okubo S, Ikezumi Y, et al. A favorable course of collagenofibrotic glomerulopathy after kidney transplantation and questionnaire survey about the prognosis of collagenofibrotic glomerulopathy. Nihon Jinzou Gakkai Shi (Japanese Journal of Nephrology) 46: 360-364, 2004 (in Japanese, Abstract in English).

5. Woodrow G, Turney JH, Davison AM, Cooper EH. Serum hyaluronan concentrations predict survival in patients with chronic renal failure on maintenance haemodialysis. Nephrol Dial Transplant 11: 98-100, 1996.

6. Stenvinkel P. High serum hyaluronan indicates poor survival in renal replacement therapy. Am J Kidney Dis 34: 1083-1088, 1999.

7. Girish KS, Kemparaju K, Nagaraju S, Vishwanath BS. Hyaluronidase inhibitors: a biological and therapeutic perspective. Curr Med Chem 16: 2261-2288, 2009.

8. Triggs-Raine B, Salo TJ, Zhang H, Wicklow BA, Natowicz MR. Mutations in HYAL1, a member of a tandemly distributed multigene family encoding disparate hyaluronidase activities, cause a newly described lysosomal disorder, mucopolysaccharidosis IX. Proc Natl Acad Sci USA 96: 6296-6300, 1999.

9. Natowicz MR, Short MP, Wang Y, et al. Clinical and biomedical manifestations of hyaluronidase deficiency. N Engl J Med 335: 1029-1033, 1996.

10. Tammi RH, Passi AG, Rilla $K$, et al. Transcriptional and posttranslational regulation of hyaluronan synthesis. FEBS J 278: 1419-1428, 2011.

11. Stridh S, Palm F, Hansell P. Renal interstitial hyaluronan: functional aspects during normal and pathological conditions. Am J Physiol Regul Integr Comp Physiol 302: R1235-R1249, 2012.

12. Kerjaschki D, Sharkey DJ, Farquhar MG. Identification and characterization of podocalyxin--the major sialoprotein of the renal glomerular epithelial cell. J Cell Biol 98: 1591-1596, 1984.

13. Nikolic-Paterson DJ, Jun Z, Tesch GH, Lan HY, Foti R, Atkins RC. De novo CD44 expression by proliferating mesangial cells in rat anti-Thy-1 nephritis. J Am Soc Nephrol 7: 1006-1014, 1996.

14. Kastner S, Thomas GJ, Jenkins RH, Davies M, Steadman R. Hyaluronan induces the selective accumulation of matrix- and cell-associated proteoglycans by mesangial cells. Am J Pathol 171: 1811-1821, 2007.

(C) 2014 The Japanese Society of Internal Medicine http://www.naika.or.jp/imonline/index.html 